\title{
Donor 24*7 using GPS Tracking System
}

\author{
Urvi Kore \\ Professor \\ Thadomal Shahani Engineering College, Mumbai, \\ India \\ P.G. Kher Marg, TPS-III, Link Road, Bandra (West) \\ Mumbai
}

\author{
Divya Pathai \\ Thadomal Shahani Engineering College, Mumbai, \\ India \\ P.G. Kher Marg, TPS-III, Link Road, Bandra (West) \\ Mumbai
}

\begin{abstract}
Emergency situations, such as accidents, create an immediate and critical need for specific blood type. In addition to emergency requirements, advances in medicine have increased the need for blood in many on-going treatments and elective surgeries. Despite increasing requirements for blood, only about $5 \%$ of the Indian population donates blood. In this paper a new and efficient way to overcome such scenarios is proposed through the executed project. Donor $24 * 7$ is an android application which can be used during emergency situations of blood requirement, you can quickly check for contacts matching a particular or related blood group and reach out to them via Phone Call/SMS through the application. This application provides list of donors in your city/area. A user-friendly application is easy to understand and use. Cloud-based services can prove important in emergency blood delivery since they can enable central and immediate access to donors' data and location from anywhere. Since almost everyone carries a mobile phone with them, it ensures instant location tracking and communication. The location-based app, operational on android platform, will help users easily find donors of matching blood groups in their location and access their mobile numbers for instant help. Only a user who is registered on the application will be able to donate or request for blood.
\end{abstract}

\section{Keywords}

Donor, Donee, Needy, Donate, Request, Android.

\section{INTRODUCTION}

Lack of blood often becomes the cause for maternal mortality and deaths in accident cases. In order to avoid any such problems and make the whole process of blood donation very efficient. DONOR $24 * 7$ provides a platform to locate a donor of a particular blood group. This will be very useful in emergency situations. This project aims in developing a platform for users which will help them to locate a donor in any emergency situation. It uses Google Maps API to detect location of a particular user. The needy will get a list of all the donors available in his/her vicinity. This makes the whole process very simple and efficient. Aiming to club the process of blood donation with technology by providing a user friendly mobile application as a smart phone is owned by almost everyone in today's time. This application will be working in a real time environment as the location of the donor can change at any point of time. Also, it's a multi-user environment. A user can request multiple users. Also, a user can get requests from multiple users to donate blood.

\section{SCOPE \& RELEVANCE}

An emergency situation can arrive at any particular time, like needing a donor of a particular blood group. Although there are blood banks, but sometimes even blood banks fail to get a particular blood group. Also, these blood banks are sometimes not sufficient. Out of approximately 16000 donations in the past 2 years, 438 tested positive for TTI, including 107 for HIV. Only $20 \%$ of the donors contacted the blood bank; none of them were HIV positive. So even if there are a lot of available donors, it is not necessary that their blood can be used and donated to someone. Bringing technology into picture, the mobile application will help any user to locate a donor in his/her vicinity. DONOR $24 * 7$ is a project aimed to make the process of finding a donor and getting a particular blood group very efficient and fast during an emergency situation. It is a smart, flexible, easy to use mobile application.

\section{EXISTING SOLUTION}

As the problem is very crucial, attempts have been made in past to solve the problem by combining it with the upcoming technology so that more lives can be saved in case of emergencies. Bloodline is one of applications used for the same. Bloodline runs algorithms to match potential blood donors in case of a request for blood. You don't need to contact donor after donor anymore, the application will do that for you and let you know if someone kind is willing to help you out. Also, the user does not get countless notifications - the algorithms used will contact you only if you're the ideal person. By signing up for Bloodline, the personal details and contact information of the user are perfectly safe - no one gets to see them unless you want them to.

\section{How Bloodline works:}

1. User signs up.

2. When we think user can make a difference by donating blood, we send you a tiny little notification. We won't bother you if you are far away, nor if you'd donated recently.

3. If a user or anyone user knows is in need of blood (say, for a surgery), just place a request. It's free.

4. We contact the relevant people - and send the user an SMS when they respond.

5. User doesn't have to call up their friends, reach out to known networks, etc. Congratulations! The user has made our community awesome!

The existing solution is user friendly and successfully automates the process of blood donation by incorporating technology. But it does not take current location in perspective. The application contacts the donors on the basis of their addresses which might take usual time. Hence it is not dynamic solution. 


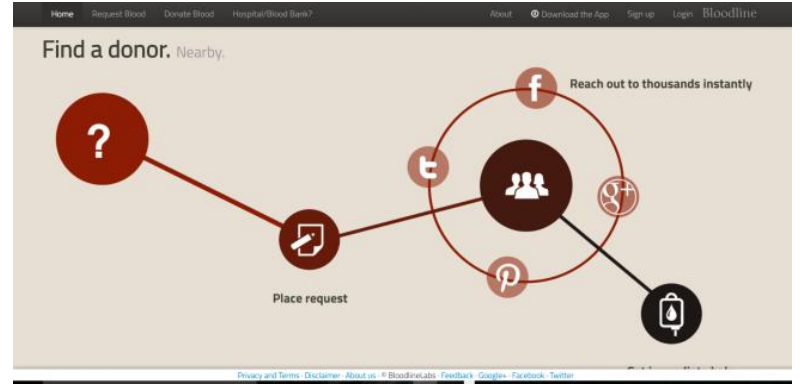

Figure 1. Bloodline Homepage

\section{PROPOSED SYSTEM}



Figure 2: Proposed System

The system is based on real time environment wherein an android based mobile application built will help the users of the application to locate a particular donor of a required blood group type in the vicinity of 10 kilometers. For developing this application we will be using Google Maps API to find the geolocation of the user's device. Both the actors- the donor and the needy, must be registered as users on the application. While registering, he/she should provide his/her basic details like name, age, gender, contact number, his/her blood group type. The needy simply needs to give the required blood group and should make sure that his/her gps is switched on. After giving information about the required blood group type, the system will generate a list of all the users with that particular blood group type in the needy's vicinity. This list will be provided to the needy.

The needy can therefore request any of the mentioned donors to donate blood. Once he/she requests a particular user to donate blood, the system will then let the donor know that a particular user has requested him/her to donate blood. It is necessary that the gps of the donor should also be switched on. As soon as a needy requests a particular user to donate blood, that user will get a notification either on application or via SMS about the request. He/She can accept the request to donate blood. Once a particular user agrees on donating his/her blood, the needy will get an in app notification that a particular donor has accepted his/her request. And then the system will provide all the information of that particular user who has agreed to donate blood in order to allow direct communication between them

\subsection{User Account}

This page is the first activity of the mobile application. In order to use this application, the user should be registered on the application. The user is asked to fill in the profile which includes details like Name, Gender, Blood Group and other contact details. After registering with the application, the user can Log In using the Email Id and Password .As soon as the user logs in, he/she is asked whether the user wants to request for blood or donate blood.

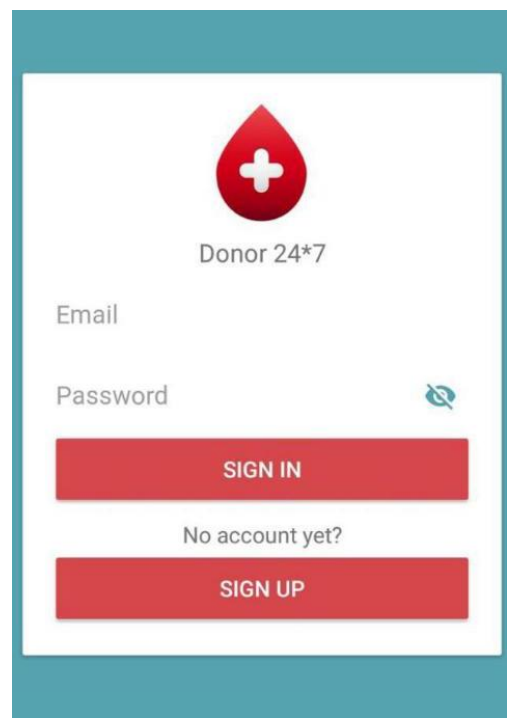

Figure 3.User Log In

\subsection{Request for a particular blood}

After Login, if the user wants to request for a particular blood, he/she is asked to select from a list of blood groups. After selecting the blood group the application takes mobile device's geolocation in terms of latitude and longitude using Google Maps API. It starts searching for all the available donors in vicinity of 10 kilometers. At the end of the search, a curated list of donors is presented to the user.

If GPS of the user is not on he/she is notified regarding the same. The user sends a particular required blood group type information to the server with mobile phone. Then the server records this information along with the information of user who has requested that particular information. After the user who has requested for a particular blood group type gives the information, the servers stores that and starts searching for a donor with the same blood group type in the vicinity of needy.



Figure 4. Requesting a blood group

\subsection{Donating Blood}

The user who selects to donate blood is first asked to answer some questions which keep a track of his health to determine whether he is fit to donate blood or no, that his blood can be 
used or no. The fitness is determined considering user's health for the past six months. Any kind of chronic disease can cause harm to the other person, hence it is necessary for all the users to answer these questions truthfully so that the purpose of this application is served. The user who is fit is allowed to donate blood otherwise if any of the conditions hold true the application does not allow the user to donate blood.


Figure 5. Fitness check

Figure 6. Unfit user notification

\subsection{Notifications and Blood}

\section{Donation}

After the list of available users is curated, an in app notification is sent to all the available donors. The notification includes needy's name, current location, contact details and the blood group required. The filtering of all the donors is done using $\mathrm{K}$ Near Algorithm. The donors are filtered according to their location and not blood group for referral purposes. The user who is registered and in the vicinity might not have the required blood group but can refer someone else who is willing to donate but not registered on the application. If according to the address mentioned in the profile, the user is nearby but there is no internet connectivity push notification is sent later when the connection is available. All the requests will be queued for that user.

The donor has two options of either accepting the request to donate blood or decline the request. The donor who is willing to donate blood can accept the request and thereby contact the needy. If there are multiple donors, the needy can choose one of his choice and start the process.

The application successfully automates the process of blood donation by using the device's geolocation services and finding the donors in the vicinity of 10 kilometers. This makes the process of finding donors quicker and more hassle-free .In order to increase the accuracy of the process, the application can be implemented at a larger scale so that more number of users are available in case of emergencies.



Figure 7. Requests

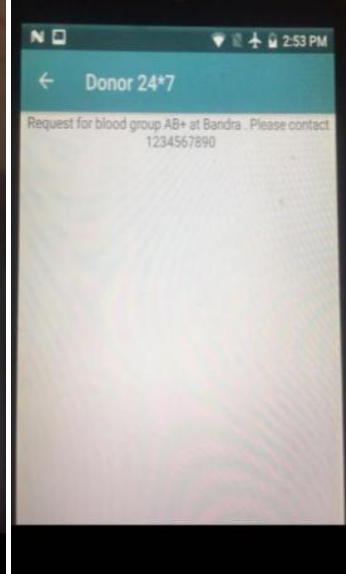

Figure 8. App Notification

\subsection{E. About Us and Help}

The About Us tab gives information about the application to the user, guiding the user about how to use the application. Help tab answers Frequently asked questions of the user. Various questions about the application as well the process of blood donation are answered. This feature makes the application better user-friendly.

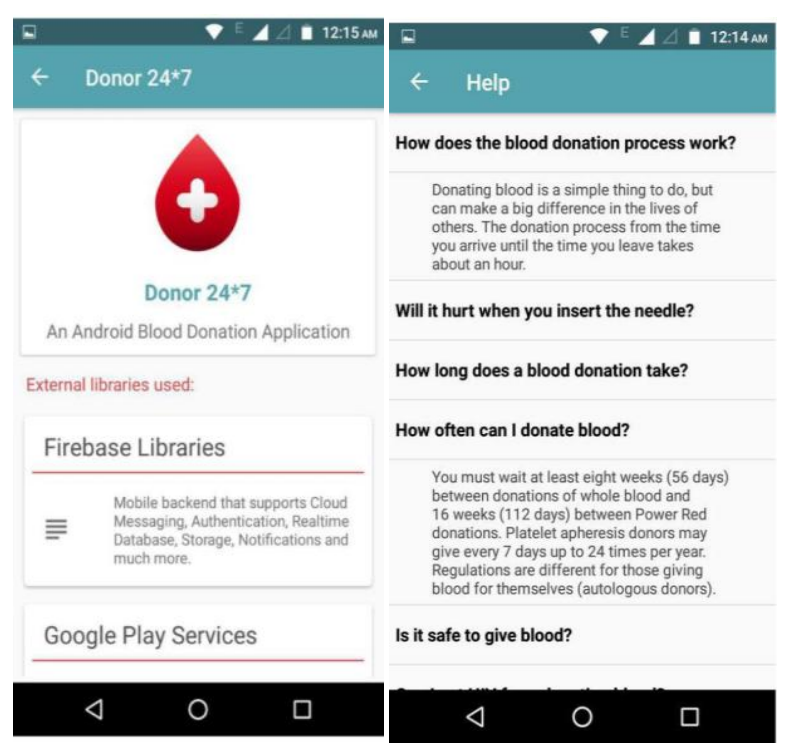

Figure 9. About Us

Figure 10. Help tab

\section{CONCLUSION}

The application being user friendly helps finding the donors quicker which is very helpful in an emergency situation. As most of the people use smart phones nowadays the application can be implemented at a higher scale increasing the availability of the donors hence making the process more accurate.

Currently the notification is sent to only users registered with the application in the vicinity of $10 \mathrm{kms}$.In the future, the application can collaborate with a hospital or a blood bank to increase the efficiency of the blood donation process on a large scale. Also, a provision for the users whose GPS is not on, making the entire process offline. Using Text Messages for notification purposes. 


\section{ACKNOWLEGEMENT}

I would like to thank Prof. Urvi Kore for her support and guidance without which I would have been unable to develop this project. I would also like to thank Dr. Tanuja Sarode, Head of the Computer Department, Thadomal Shahani Engineering College and Dr. G. T. Thampi, Principal of Thadomal Shahani Engineering College for their support.

\section{REFERENCES}

1. Kumbhar, A. A., Wanjara, K. S., Trivedi, D. H., Khairatkar, A. U., \& Sharma, D. (2014). "Automated Attendance Monitoring System using Android Platform.”, International Journal of Current Engineering and Technology, Vol. 4, No. 2, pp1096-1099.
2. Bandra.U,Bandra.P,"Tagciti:A practical approach for location-aware and socially relevant information creation and discovery for mobile users,"IEEE International symposium on Wireless communication systems,Reykjavik,pp.118-122,2008.

3. Gupta.A.kumar,S.Qadeer,M.A.,'Location based services using android(LBSOID)," IEEE International conference on Multimedia services architecture and applications,pp $1-5,2009$.

4. Bloodline-A Network for Life bloodlinelabs.com1

5. Markers $\mid$ Google Maps Android API $<$ https://developers.google.com/maps/documentation /an droid-api/marker\#code_samples>Web. 24 Feb. 2016. 